\section{Massive Serous Retinal Detachment and Acute Intraoperative Rock-hard Eye Syndrome after Cataract Surgery: A Case Report}

\section{Dear Editor,}

Serous retinal detachment (RD) after uncomplicated cataract surgery is rare. Recently, the number of reports on this condition has been increasing because of the popularity of intracameral cefuroxime injections [1]. We recently treated a woman with primary angle-closure glaucoma (PACG) who had a serous RD after uncomplicated cataract surgery.

A 62-year-old Korean female patient underwent cataract surgery in her left eye. Four years prior to cataract surgery, she underwent bilateral laser peripheral iridotomies. She had not been diagnosed with any chorioretinal disease and preoperative fundus examination was normal. After phacoemulsification, at the beginning of irrigation and aspiration, the anterior chamber collapsed. The eyeball was very hard with digital palpation. There was no sign of suprachoroidal hemorrhage including loss of the red reflex or severe pain. The surgeon presumed this situation to be acute intraoperative rock-hard eye syndrome (AIRHES) and performed retrolenticular aspiration via pars plana with a $25 \mathrm{G}$ needle. Using this procedure, the eyeball was softened. At the end of the surgery, $0.01 \%$ carbachol was injected into the anterior chamber. Unexpectedly, the anterior chamber became shallow. Immediately postoperatively, a $1 \%$ atropine eye drop was instilled. Postoperative topical antibiotics and steroid eye drops were administered.

Five days after surgery, the uncorrected visual acuity was $20 / 50$, and the intraocular pressure (IOP) was 17 $\mathrm{mmHg}$ with $2 \%$ carteolol; however, the patient complained of photopsia in the left eye. A fundus examination revealed

Received: April 15, 2021 Final revision: November 27, 2021 Accepted: November 29, 2021 a localized serous RD (Fig. 1A). Three weeks after surgery, the RD had expanded to involve the macula. It continued to expand until 5 weeks after surgery (Fig. 1B, 1C). Fluorescein angiography and indocyanine green angiography, which were performed 5 weeks postoperatively, revealed a pinpoint leakage near the retinal pigment epithelial detachment region. Therefore, focal laser treatment was performed at the leaking point. One month after the laser treatment, the serous RD had resolved (Fig. 1D).

AIRHES is characterized by acute intraoperative shallowing of the anterior chamber and a marked increase in intraocular pressure with the absence of choroidal hemorrhage or effusion. AIRHES is also called infusion misdirection syndrome, due to the fact that the latter's pathophysiology is inappropriate movement of the irrigating fluid via the zonular fibers [2]. Vitreous tap was performed to relieve AIRHES and was effective in the present case.

Chang et al. [3] reported acute uveal effusion with preoperative central serous chorioretinopathy (CSCR) during phacoemulsification. Their case and ours shared similarities: cataract surgery was performed in eyes with PACG, and AIRHES developed. In the former case, however, dry pars plana vitrectomy was performed to relieve the AIRHES and uveal effusion rather than the aggravation of CSCR developed postoperatively. In contrast to their case, our patient had no chorioretinal lesion preoperatively, received a vitreous tap, and had a massive serous RD that resembled CSCR postoperatively. A pars plana vitreous tap might have less effect on IOP than pars plana vitrectomy as it could remove a smaller amount of retrolenticular fluid. In this light, the lesser intraoperative IOP fluctuation in our patient may have induced serous RD rather than uveal effusion. Vitreous tap might change IOP abruptly, breakdown of the blood-ocular barrier already induced by cataract surgery could progress further resulting in choroidal expansion. Choroidal expansion, which has recently been considered to be one of the major mechanisms related to PACG, can also cause serious RD [4].

The IOL-iris diaphragm was displaced anteriorly again after intracameral carbachol injection in our case which could induce vitreous traction resulting in serous RD. Miotic-induced RD is usually rhegmatogenous RD because vitreous traction caused by miotics can make new retinal 

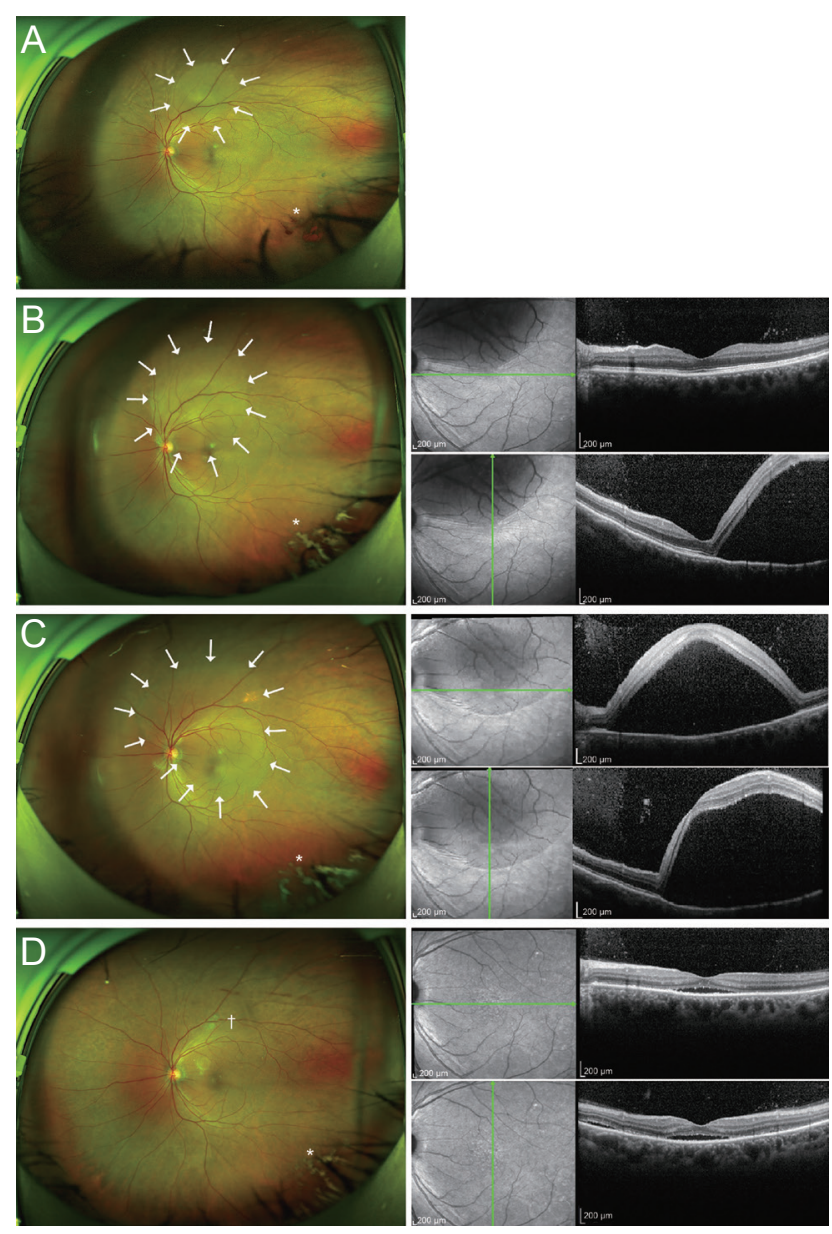

Fig. 1 Serial wide-field photography (left) and optical coherence tomography (right) images of the left eye. White arrows indicate the extent of serous retinal detachment (RD). (A) The wide-fundus photo taken at 5 days after cataract surgery shows localized serous RD and minimal vitreous hemorrhage (asterisk). (B) Serous RD expanded to threaten the macula at 2 weeks after cataract surgery. (C) Serous RD continued to expand until 5 weeks after cataract surgery when focal laser treatment was performed. (D) Serous RD resolved after focal laser treatment. Resolving vitreous hemorrhage (asterisk) and laser burns (dagger) are also shown.

breaks or act on preexisting retinal breaks. Another possible mechanism is miotic therapy, which might weaken the adhesion between the retinal pigment epithelium and the photoreceptors [5]. When subretinal fluid is derived from the vessels of the choroid, weak adhesion between the retinal pigment epithelium and the photoreceptors might promote accumulation of the fluid.

In summary, our patient was diagnosed with PACG pre- operatively, which itself is a risk factor for AIRHES. During the surgery, a pars plana vitreous tap and intracameral carbachol injection were performed. Both of these procedures could cause serious RD after uncomplicated cataract surgery. To the best of our knowledge, this is the first study to report on a case of both AIRHES and postoperative serous RD.

Min-Ji Kang ${ }^{*}$, Je Hyung Hwang*, Jee Hye Lee, Ha Eun Sim, Won Hyuk Oh

Department of Ophthalmology, Sanggye Paik Hospital, Inje University College of Medicine, Seoul, Korea E-mail(Won HyukOh): briol7@naver.com

"Min-Ji Kang and Je Hyung Hwang contributed equally to this work.

\section{Conflicts of Interest}

No potential conflict of interest relevant to this article was reported.

\section{Acknowledgements}

None.

\section{Funding}

The authors received no financial support for this article.

\section{References}

1. Zuo C, Mi L, Ye D, et al. Toxic retinopathy after phacoemulsification when the cefuroxime dilution is correct. $J$ Cataract Refract Surg 2018;44:28-33.

2. Grzybowski A, Prasad S. Acute aqueous misdirection syndrome: pathophysiology and management. J Cataract Refract Surg 2014;40:2167.

3. Chang R, Du Y, Peng Z, et al. Acute uveal effusion during phacoemulsification with preoperative central serous chorioretinopathy: a case report. BMC Ophthalmol 2017;17:137. 
4. Soubrane-Daguet G, Coscas G. Serous and hemorrhagic detachment of the sensory retina and pigment epithelium. In: Schachat AP, Sadda SR, Hinton DR, et al., editors. $R y$ an's retina. 6th ed. Amsterdam: Elsevier; 2018. p. 703-10.
5. Kraushar MF, Steinberg JA. Miotics and retinal detachment: upgrading the community standard. Surv Ophthalmol 1991;35:311-6. 